\title{
Modeling of Vortex Induced Vibration based Hydrokinetic Energy Converter
}

\author{
Krishna Manasa Rao ${ }^{1}$ and Ashray Gururaja Manur $^{2}$ \\ ${ }^{1,2}$ Department of Electrical and Electronics Engineering, Sri Jayachamarajendra College of Engineering, India
}

\begin{abstract}
Vortex Induced Vibrations (VIV) forms the basis of the hydrokinetic energy converter. With the increasing energy demand, there is a need for a significant shift to using clean and renewable sources of energy. VIV based hydrokinetic energy converter aims to harness aquatic energy from slow moving water currents. For modeling, a single cylinder system with single degree-of-freedom is considered. This paper deals with formulation of generalized equations to characterize the flow oscillator interaction and to develop a model to carry out experimental observations of the hydrokinetic energy converter. The model will help predict the dynamic response and ultimately determine the power that can be extracted. The kinetic energy of the fluid flow produces transverse mechanical motion of the bluff body. This mechanical motion is then used to produce useful electrical energy with the help of a Power Take Off (PTO) system. The theoretical maximum energy that can be harnessed by the PTO has also been discussed.
\end{abstract}

Keywords: Hydrokinetic Energy Converter, Kutta- Joukowski Theorem, Reynolds Number, Strouhal Number, Vortex Induced Vibrations

\section{INTRODUCTION}

VIV is a complex fluid-structure phenomenon. Most of the studies in this area have been carried out in order to minimize the fatigue on the structures caused by VIV. However, recent energy studies related to VIV has been aimed to enhance the vibrations in order to maximize energy extraction from fluid flow [1]. VIV based hydrokinetic energy converter has several advantages over conventional hydrokinetic energy converters which are:

- Ability to harness energy from water flow with a flow velocity as low as $0.25 \mathrm{~m} / \mathrm{s}$

- High energy density

- Less likely to cause ecological imbalance

- Robust and cost effective

- Less maintenance cost

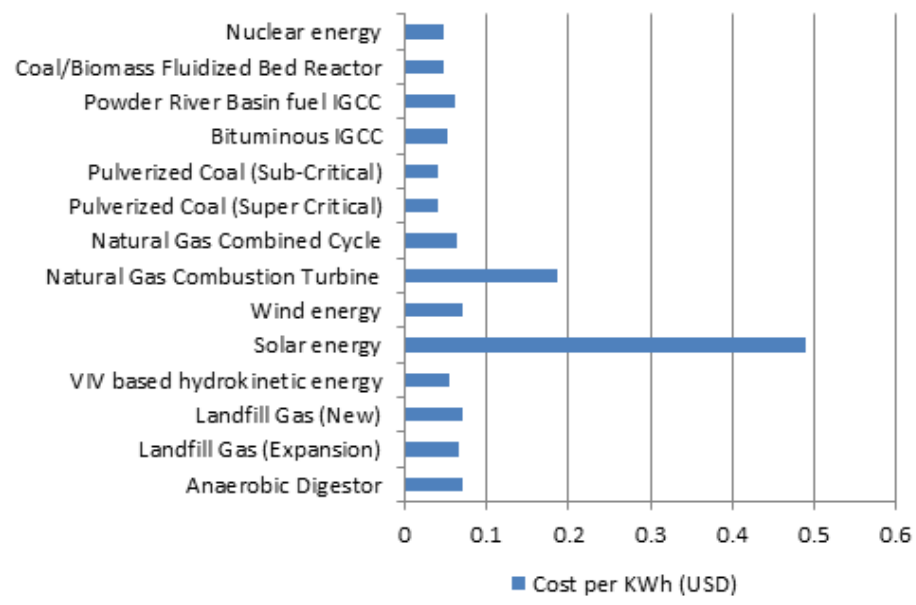

Figure1: Cost comparison of various energy sources [2]

\section{NON-LINEARITY IN VIV}

Vortex Induced Vibration (VIV) is a highly non-linear phenomenon. When the frequency of vortex shedding $f_{s}$ is close to the natural frequency of the body in motion $f_{n}$, the phenomenon of lock in occurs. The range of frequencies over which lock in occurs is one of the most important areas of research. Lock in is a nonlinear phenomenon where in, the highest amplitude is not observed exactly at the point where the shedding 
frequency equals the natural frequency. It occurs over a range and is self-limiting in nature where, as the amplitude of the bluff body increases, the VIV is suppressed which in turn affects the motion of the bluff body.

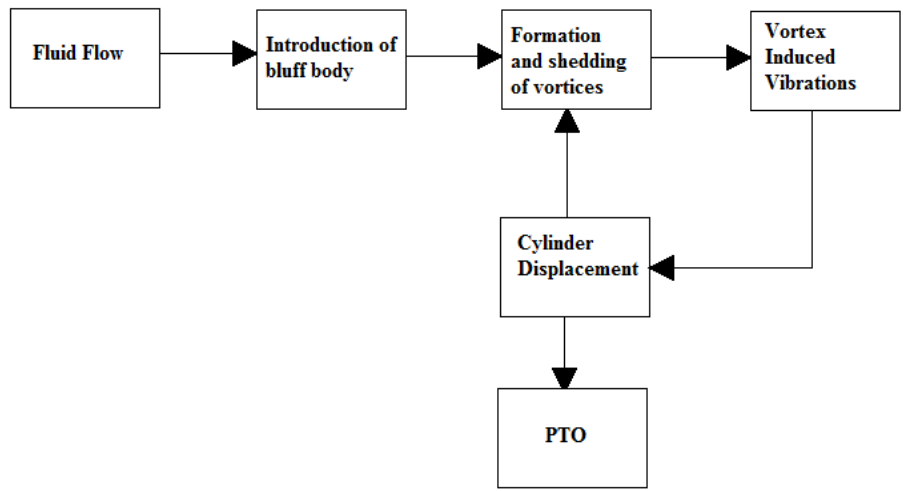

Figure 2: Basic working principle of VIV based hydrokinetic energy converter

\section{MATHEMATICAL MODEL}

There are several parameters either in the form of constants or variables which have to be evaluated in order to model this hydrokinetic energy converter. The natural frequency of the spring-cylinder system is given by

$$
f_{n=\frac{1}{2 \Pi}} \sqrt{\frac{k}{m_{\text {app }}}}
$$

In the equation above, $f_{n}$ is the natural frequency of the spring-cylinder system, $k$ is the spring constant and $m_{a p p}$ is the apparent mass of the cylinder. Since the cylinder is submerged in the water, its weight cannot be directly taken for calculation. Its apparent weight as per Archimedes principle has to be considered which is given as

$$
m_{\text {app }}=m_{o b j}-m_{\text {dis }}
$$

where $m_{o b j}$ is the real weight and $m_{d i s}$ is the weight of the fluid displaced.

$$
m_{\text {dis }}=\rho_{\text {fluid }} * v_{\text {o }} l_{\text {cyl }}
$$

The natural frequency of the system is important in determining the range in which lock in is likely to occur. Range over which lock in occurs also depends on mass ratio $m^{*}$ which is defined as

$$
m^{*}=\frac{m_{\text {dis }}}{m_{\text {osc }}}
$$

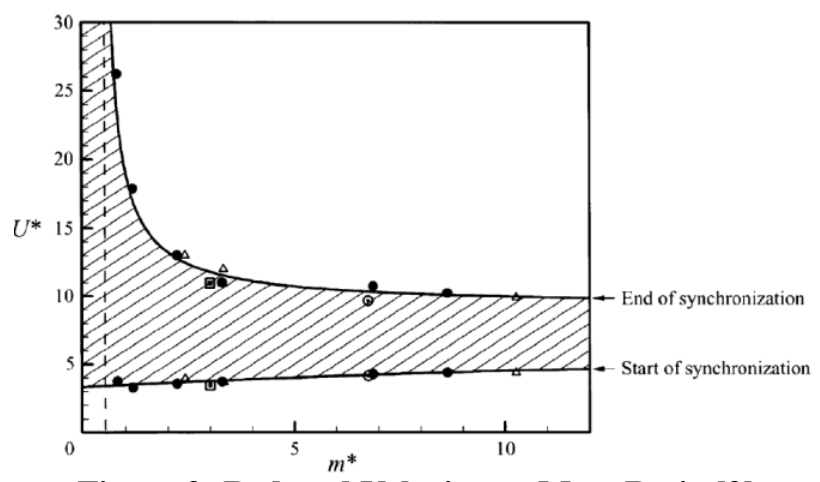

Figure 3: Reduced Velocity vs. Mass Ratio [3]

$U^{*}$ is a non-dimensional parameter known as reduced velocity. It is used in experimental analysis for measuring amplitude vibration and is given by

$$
U^{*}=\frac{U}{f_{n} D}
$$

For lower values of $m^{*}$, the upper limit of the lock in begins to grow exponentially, but for lower values the variation is insignificant.

Reynolds number is one of the most important parameter which describes the structure-fluid interaction. It is defined as the ratio of inertial forces to viscous forces and is given by

$$
R e=\frac{U d}{v}
$$


Where $U$ is the velocity of free stream, $d$ is the characteristic length and $v$ is the kinematic viscosity.

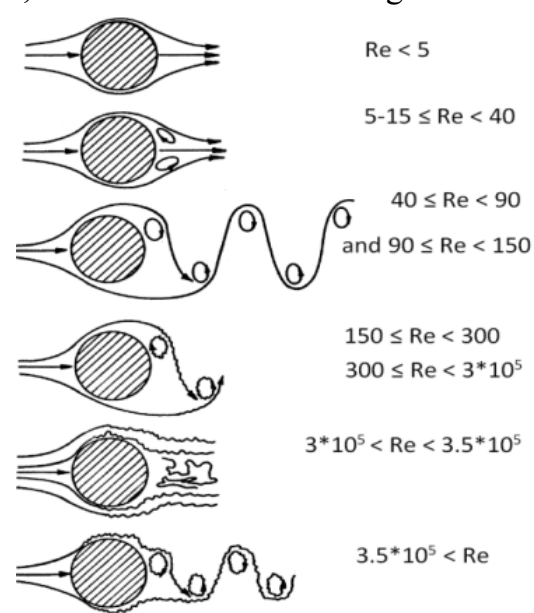

Figure 4: Regimes of fluid flow across cylinders [4]

For effective working of the hydrokinetic energy converter in discussion, dead zones should be avoided as the net lift force generated due to VIV is negligent or nil. In lower regimes, the fluid flow is laminar which is not useful for energy generation. As the Reynolds number is increased, a laminar to turbulent transition takes place in the vortices. In the range of $10^{5}<R e<5^{*} 10^{5}$, the laminar boundary on the cylinder becomes turbulent. Another important non-dimensional parameter used to describe oscillating flow conditions is Strouhal number. It relates the frequency of vortex shedding to the flow conditions and is given by

$$
S=\frac{f_{S} D}{U}
$$

where $f_{s}$ is the frequency of vortex shedding, $D$ is the diameter of the cylinder and $U$ is the fluid flow velocity. For large Strouhal numbers, viscosity dominates fluid flow, resulting in a collective oscillating movement of the fluid. For low Strouhal numbers (order of $10^{-4}$ and below) the high-speed quasi steady-state portion of the movement dominates the oscillation. Oscillations at intermediate Strouhal numbers are characterized by buildup and rapidly subsequent shedding vortices. The vortex shedding frequency is evaluated from the above equation on the rough assumption that Strouhal number has a constant value of 0.21 [4]. The lift force is another important parameter which plays a vital role in determining the final energy output. The KuttaJoukowskitheorem can be used to determine the quantity of lift on the bluff body due to fluid flow. It is given by

$$
\begin{aligned}
& m \ddot{y}+c \dot{y}+k y=L \\
& L=\rho U \Gamma
\end{aligned}
$$

$\rho$ is the density of fluid, $U$ is the velocity of the fluid and $\Gamma$ is defined as

$\Gamma=\oint U \cdot d s$

The line integral is around a contour in anti-clockwise direction enclosing the cylinder such that the path is in the region of potential flow and not in the boundary layer of the cylinder. Linear equations for amplitude of oscillation and lift coefficient can be written as

$y=y_{\max } \sin \left(2 \Pi f_{s} t\right)$

$c_{L}(t)=C_{L} \sin \left(2 \pi f_{s} t+\varphi\right)$

In the above two equations, $y_{\max }$ is the maximum amplitude of oscillation, $\varphi$ is the phase angle between fluid force and displacement, $c_{L}$ is time independent lift coefficient and $C_{L}$ is the amplitude of lift coefficient of the cylinder.The power in the fluid which is flowing over the cylinder whose motion is perpendicular to the fluid flow is given by

$$
\text { Powerinfluid }=\frac{1}{2} \rho U^{2} * D L
$$

Here $\rho$ is the density of the fluid, $D$ is the diameter of the cylinder, $U$ is the stream velocity and $L$ is the length of the cylinder.The work done by the fluid acting on the bluff body during a vortex induced vibration cycle is given by

$$
W_{V I V}=\int_{0}^{T_{c y l}} F_{\text {fluid }} \dot{y} d t
$$

The fluid power due to VIV is

$$
P_{V I V}=\frac{W_{V I V}}{T_{c y l}}
$$


To obtain $P_{V I V}$, we multiply the force from (8) with the instantaneous velocity and perform the integration as above to obtain

$$
P_{V I V}=\frac{1}{2} \rho C_{L} f_{s} y_{\max } D L \sin (\varphi)
$$

The efficiency of the system can then be given as

$$
\eta_{V I V}=\frac{P_{V I V}}{\text { Powerint hefluid }}
$$

From (13) and (16) we have

$$
\eta_{V I V}=\frac{\frac{1}{2} \rho C_{L} f_{s} y_{\max } D L \sin (\varphi)}{\frac{1}{2} \rho U^{2} * D L}
$$

\section{MODELING OF PHYSICAL FEATURES OF FLUID FLOW}

There are several physical features which directly affect the mathematical model.

- Stagnation point

- Separation point

- Formation point

- Boundary layer

- Shear layer

The stagnation point $\theta_{s t}$ is the point of zero velocity on the body. In viscous flow, it is defined as the point of highest pressure. The stagnation point moves with the motion of the body as well as the formation and shedding of the vortices. The amplitude of motion of the stagnation point is denoted by $\Theta_{s t}$ and the relation between the stagnation point and amplitude of motion of stagnation point is given by

$\theta_{s t}=\Theta_{s t} f(t)$

The separation point is defined as the point where the wall shear stress is exactly zero or the point where fluid particles leave the wall. A strong relationship between separation point and Reynolds number has been established.

$$
\theta_{s p}=\Theta_{s p} f(t)
$$

Formation point is a point in the near wake at which the shear layer saturates and begins to form the vonKármán vortex. It moves away from the body during the formation of the vortex and quickly snaps back to the body during the shedding of the vortex. Denoted by $x_{f p}$, it is highly dependent on $R e$ and experiments have been carried out to prove the dependence of formation point on the Reynolds number [5]. The boundary layer exists between the stagnation point and each respective separation point. It is highly dependent on $R e$ since viscous forces dominate in this region. The shear layer exists between the separation point and the formation point. The shear layer is also known as the feeding layer in the case of VIV since it feeds energy into the vortex.

\section{MODELING OF DYNAMIC RESPONSE OF BLUFF BODY}

Dynamic response of a bluff body in fluid flow incorporates finding the amplitude of vibration of the cylinder. This has been one of the most crucial tasks of mathematical modeling. In the hydrokinetic energy converter, the bluff body is supported by a spring system which aids its movement. The amplitude of vibration can be found out practically. However, arriving at a range of values for the amplitude is theoretically complicated and often not very effective because several factors such as losses in vibration owing to spring action and viscosity are not taken into consideration. When a cylindrical bluff body is moving in water, the amplitude envelope is described by a decaying exponential function which is given by

$$
y=a e^{\sigma x}
$$

where $a$ is the amplitude factor and $\sigma$ is the product of damping coefficient $\chi$ and $\omega_{n}$, which is the natural frequency of the system while $x$ is the vortex shedding frequency. The above equation is a Gaussian equation for finding the amplitude of a system immersed in water. Envelopes are used to control the way vortices change over time. The amplitude envelope of a vortex, when a cylindrical bluff body is used can be given by

$y=\cos (2 x) e^{-0.05 x}$

where $\mathrm{x}$ is the vortex shedding frequency.

$\ln (y)=\ln (a)+\sigma x$

In order to calculate the values of $a$ and $\sigma$ we optimize the following

$$
Y=A+B X
$$


Using piece-wise linear minimization we obtain

$$
\begin{gathered}
A=\left[\begin{array}{cc}
1 & x 1 \\
\vdots & \vdots \\
1 & x n
\end{array}\right] \\
B=\left[\begin{array}{c}
b 1 \\
\vdots \\
b n
\end{array}\right] \\
p=\left[\begin{array}{c}
a \\
\vdots \\
\sigma
\end{array}\right]
\end{gathered}
$$

where $p$ is the optimized solution. The amplitude is then calculated using the formula

amplitude $=p e^{\sigma x}$

For simulating the working of VIV based hydrokinetic energy converter, it is essential to create artificial river flow conditions. This requires large channels of flowing water involving multi-layer fluid flow. To make the experimental setup cost-effective, a recirculating tank was manufactured. Recirculating flow was established with the help of central channel and flow guide vanes. In the absence of recirculating fluid flow, the dynamic response of the bluff body obtained in MATLAB is shown in fig 5.

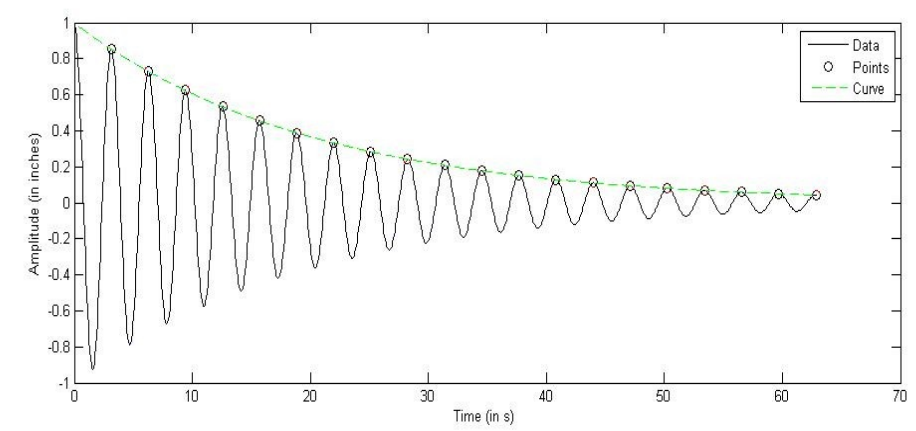

Figure 5: Dynamic Response of bluff body in fluid flow

\section{ELECTRIC POWER OUTPUT OF PTO}

The PTO of the hydrokinetic energy converter is a linear generator [6]. It is primarily based on Faraday's laws of electromagnetic induction.

$$
\begin{aligned}
& E=N \frac{d \phi}{d t} \\
& \phi_{B}=B A(\cos \theta)
\end{aligned}
$$

$B$ is the magnetic field strength, $A$ is the area and $\theta$ is the angle between $B$ and normal to the area $A$. The two factors $A$ and $B$ depend on the magnet design and coil optimization. The EMF produced will depend on number of turns of wire and rate of change of flux. The rate of change of flux depends on the motion of the bluff body. Although the speed of the cylinder changes continuously, in order to simplify the calculations, the average speed of the cylinder which is equal to $v$ was taken. The rate of change of flux is now given by

$$
\frac{d \phi_{B}}{d t}=\frac{\phi_{B}}{\frac{h}{v}}
$$

wherehis the height of the winding. The core-coil system in the linear generator employs single magnet and multiple-coil assembly. The number of turns on the iron core is given by

$$
N=\frac{r_{c}-a}{g} * \frac{w_{n}}{g}
$$

$r_{c}$ is the core diameter including the windings, $w_{n}$ is the width of the notch and $g$ is the gauge of the wire. The calculation for the length of the copper wire that should be obtained for $N$ number of turns is fairly complicated. This is because even though the length of one turn is equal to the circumference of that layer, each subsequent layer will increase the radius of the core with the coil.

$$
\text { lengthof copperwire lneeded }=\frac{w_{n}}{g} * \sum_{n=0}^{n=\frac{r_{c}-a}{g}} 2 \Pi *(a+n g)
$$

The length of the copper wire directly affects the inductance of the system and the inductance of a coil with an air core is given by

$$
L_{0}=\frac{N^{2} r}{6 r+9 l+10 d}
$$


$N$ is the number of turns, $l$ is the length of the wire, $d$ is the thickness and $r$ is the radius of the copper wire. However, an iron core is used in the linear generator and the inductance of an iron core system is given by

$$
L=L_{0} * \frac{\mu}{\mu_{0}}
$$

$\mu$ is the permeability of the core and $\mu_{0}$ is the permeability of air. High inductance increases the time constant of the electrical system. It is given by

$$
\tau=\frac{L}{R}
$$

For a generator to produce electrical power output it must contain a closed circuit. The coil system of the linear generator itself is not a closed circuit. The linear generator system essentially is a RL circuit and its output is

$I=\frac{V}{R} *\left(1-e^{-\left(\frac{t}{\tau}\right)}\right)$

$P(t)=V I$

\section{SIMULATION RESULTS}

Table 1: Simulation results obtained in MATLAB

\begin{tabular}{|l|l|l|l|l|}
\hline $\begin{array}{l}\text { Diameter of } \\
\text { cylinder (in m) }\end{array}$ & $\begin{array}{l}\text { Length of the } \\
\text { cylinder (in m) }\end{array}$ & $\begin{array}{l}\text { N (number of } \\
\text { cylinders) }\end{array}$ & $\begin{array}{l}\text { U (velocity of } \\
\text { stream) }\end{array}$ & $\begin{array}{l}\text { Output Power of } \\
\text { PTO (MW) }\end{array}$ \\
\hline 0.5 & 1 & 20 & 0.25 & 0.0767 \\
\hline 0.5 & 1 & 20 & 0.4 & 0.1552 \\
\hline 0.5 & 1 & 50 & 0.3 & 0.4019 \\
\hline 0.5 & 50 & 0.45 & 0.663 \\
\hline 0.5 & 1 & 500 & 0.75 & 0.9498 \\
\hline 0.5 & 1 & 20 & 0.5 & 1.2537 \\
\hline 0.5 & 5 & 50 & 0.66 & 1.6931 \\
\hline 1 & 5 & 50 & 0.5 & 2.0505 \\
\hline 2 & 5 & 200 & 0.3 & 8.1197 \\
\hline 2 & 5 & 500 & 0.5 & 9.6421 \\
\hline 2 & 5 & 200 & 0.3 & 37.0376 \\
\hline 2 & 10 & 900 & 0.75 & 88.1188 \\
\hline
\end{tabular}

\section{CONCLUSION}

In this paper, a new mathematical model has been developed taking into consideration all parameters that are physically meaningful and experimentally measurable. The model involves non-linearity and acylinder system with single degree-of-freedom. A detailed analysis of the dynamic response has been presented, keeping in mind energy extraction as paramount importance. It is extremely important to harness the energy available due to VIV and hence modeling of PTO plays an important role in the overall energy extraction. The simulation results illustrate that VIV based hydrokinetic energy converter has great potency in the field of green renewable energy.

\section{ACKNOWLEDGEMENTS}

The authors would like to thank Mrs. G M Rekha and Mrs.Kamalini R for funding this project and for their constant enthusiasm and encouragement. Special thanks to Ms.Anusha Gururaja Manur and Ms.Manisha Krishna Rao who have been a pillar of support and without whose help this project could not have been successfully completed.

\section{REFERENCES}

[1] M.M. Bernitsas, J. D. Allan, P. W. Webb, and T. Lyon, "Feasibility assessment of the VIVACE ocean/river current energy converter,'Technical report, The University of Michigan Graham Environmental Sustainability Institute, 2006

[2] M. M. Bernitsas, K. Raghavan, Y. Ben-Simon, and E. M. H. Garcia, "VIVACE (Vortex Induced Vibration for Aquatic Clean Energy): A new concept in generation of clean and renewable energy from fluid flow,"Journal of Offshore Mechanics andArctic Engineering, vol. 130,2008

[3] C.H.K.Williamson and R. Govardhan, "Vortex-Induced Vibrations," Annual Review of Fluid Mechanics, vol. 36, pp. 413-455, January 2004.

[4] John H. Leinhard, Synopsis of Lift, Drag, and Vortex Frequency data for Rigid Cylinders ,Washington State University, 1966

[5] A. Prasad and C. H. K. Williamson, "The instability of the shear layer separation from a bluff body," Journal of Fluid Mechanics 333, 375-402, 1997

[6] Krishna Manasa Rao and Ashray Gururaja Manur, "Design Optimization of Linear Generator for a Hydrokinetic Energy Converter," IOSR Journal of Electrical and Electronics Engineering (IOSR-JEEE), Volume 6, Issue 1, PP 37-40, 2013 